\title{
Evaluación de los riesgos químicos por inhalación de las sustancias utilizadas en una industria gráfica
}

\section{Evaluation of chemical risks by inhalation of substances used in a graphic industry}

\section{Wendy Villalobos-González ${ }^{1}$, José Pablo Sibaja-Brenes², José Carlos Mora-Barrantes ${ }^{3}$, Benjamín Álvarez-Garay ${ }^{4}$}

Villalobos-González, W; Sibaja-Brenes, J.P;

Mora-Barrantes, J.C; Álvarez-Garay, B. Evaluación de los riesgos químicos por inhalación de las sustancias utilizadas en una industria gráfica. Tecnología en Marcha. Vol. 34-2. Abril-Junio 2021. Pág 122-136.

doi) https://doi.org/10.18845/tm.v34i2.4977

1 Investigadora y académica, Universidad Estatal a Distancia (UNED), Costa Rica. Correo electrónico: wvillalobosg@uned.ac.cr.

(iD) https://orcid.org/0000-0002-3660-0169

2 Investigadora y académico, Laboratorio de Química de la Atmósfera (LAQAT), Universidad Nacional (UNA), Costa Rica.

Correo electrónico: jose.sibaja.brenes@una.cr.

(iD https://orcid.org/0000-0002-7056-2717

3 Investigadora y académico, Laboratorio de Química de la Atmósfera (LAQAT), Universidad Nacional (UNA), Costa Rica.

Correo electrónico: jose.mora.barrantes@una.cr.

(D) https://orcid.org/0000-0002-0409-5276

4 Investigadora y académica, Universidad Estatal a Distancia (UNED), Costa

Rica. Correo electrónico: balvarez@uned.ac.cr.

(iD https://orcid.org/0000-0002-2180-0870 


\title{
Palabras clave
}

Industria gráfica; riesgo químico por inhalación; identificación; valorización; priorización.

\section{Resumen}

En el proceso productivo de una industria gráfica, se utilizan insumos y materias primas para la impresión de documentos. Un insumo son los productos químicos que podrían generar un riesgo químico, si no se controlan de forma adecuada mediante protocolos de uso, almacenamiento y disposición de desechos. Algunas sustancias químicas utilizadas son: tintas, disolventes, diluyentes, pegamentos, gomas protectoras, reveladores de plancha, entre otros. El riesgo químico por inhalación (RQI) es generado en los lugares de trabajo por el uso de sustancias con cantidades de compuestos orgánicos volátiles (VOCs), que podrían afectar negativamente la salud de los trabajadores, una evaluación de RQI podría contribuir a identificar y evaluar las sustancias químicas y generar medidas para la disminución en el impacto a la salud. Estas evaluaciones involucran medir factores como: la cantidad utilizada de una sustancia química, la exposición que tiene una persona con el contaminante en el medio y la toxicidad del producto químico. En el presente proyecto de investigación se realizó una identificación, valorización y priorización del nivel de riesgo químico por inhalación (NRQI) generados en una industria litográfica tipo "offset" en Costa Rica. La identificación de los riesgos y peligros se efectuó utilizando herramientas como: revisión bibliográfica, lista de chequeo, entrevistas e inspecciones de campo. La valorización y priorización de los NRQI se llevó a cabo mediante la metodología francés del Instituto Nacional de Investigación en Seguridad (INRS). Del total de los 15 productos químicos utilizados por la industria, se obtuvo que el 53,3 \% presentan un RQI muy elevado. Los productos que presentan el mayor valor de NRQI son: el thinner y el limpiador de rodillos, los cuales se utilizan para la limpieza de las máquinas con residuos de tinta, con una frecuencia de uso continua en cantidades entre los $0,7 \mathrm{~L} /$ mes y los $20,0 \mathrm{~L} / \mathrm{mes}$. Por el contrario, se identificaron cinco productos con valores bajos de NRQI, tales como: la tinta en cartucho, el refrigerante, el limpiador 4 , el isopropanol y la goma protectora de planchas.

\section{Keywords}

Graphic industry; chemical inhalation risk; identification; recovery; prioritization.

\begin{abstract}
In the production process of a graphic industry, inputs and raw materials used for printing documents. An input are chemical products that could generate a chemical risk, if not properly controlled through protocols for use, storage and disposal of waste. Some chemical substances used: inks, solvents, thinners, glues, protective rubbers, plate developers, among others. Chemical inhalation risk (RQI) generated in the workplace using substances with amounts of volatile organic compounds (VOCs), which could adversely affect the health of workers. A chemical risk assessment involves evaluating factors such as: the amount of a chemical, the exposure of a person with the contaminant in the environment and the toxicity of the chemical; its purpose is to identify and evaluate chemical substances and generate measures to reduce the impact on health. In the present research project an identification, valorization and prioritization of the level of chemical risk by inhalation (NRQI) generated in a lithographic offset industry in Costa Rica carried out. The identification of risks and dangers carried out using tools such as: literature review, checklist, interviews and field inspections. The valuation and prioritization of the RQI carried out using the French methodology of the National Institute for Security Research (INRS). Of the 15 chemical products used by the industry, it obtained that $53.3 \%$ have a very
\end{abstract}


high RQI. The products with the highest NRQI value were the thinner and the roller cleaner, which used for cleaning machines, with a frequency of continuous use in quantities between 0,7 $\mathrm{L} /$ month and 20,0 L/month. On the contrary, five products with low NRQI values identified, such as: ink in cartridge, coolant, cleaner 4 , isopropanol and plate gum.

\section{Introducción}

La industria gráfica es una actividad comercial dedicada a la impresión de documentos con diferentes sistemas tecnológicos y diversos procesos de impresión [1]. Un proceso litográfico generalmente se divide en tres etapas: pre-impresión, impresión y post-impresión. En la pre-impresión se incluye las actividades de diseño, diagramación, montaje y autoedición, transfiriéndose el diseño a un portador de imágenes (plancha). En la impresión, la plancha se coloca en la impresora y en la post-impresión se incluyen los acabados finales de los materiales para comercialización. Estas operaciones involucran procesos físicos y/o químicos [2].

En la industria litográfica como en cualquier otra industria de carácter químico pueden existir presencia de agentes físicos, biológicos y químicos en el ambiente laboral con efectos sobre la salud de los trabajadores [3]. La industria gráfica utiliza sustancias químicas como materia prima e insumos para el proceso productivo, que pueden generar peligros y riesgos químicos, que repercuten en la salud de los trabajadores. Dentro de las sustancias químicas utilizadas destacan: productos de preimpresión (reveladores), disoluciones diluyentes de tintas, disolventes limpiadores, tintas, adhesivos y pegamentos [2]. Un uso inadecuado de materias primas y sustancias químicas en la industria gráfica aumenta el riesgo químico y por lo tanto el efecto sobre las personas [4]. Los disolventes y limpiadores utilizados en procesos litográficos se componen principalmente de compuestos orgánicos volátiles (VOCs, por sus siglas en inglés). En el cuadro 1, se muestran algunos efectos de los disolventes que contienen VOCs según tiempo y forma de exposición.

Cuadro 1. Efectos de los VOCs en el organismo, según método de entrada y tiempo de exposición.

\begin{tabular}{|c|c|c|}
\hline Método de exposición & $\begin{array}{c}\text { Tiempo de } \\
\text { exposición }\end{array}$ & Efectos \\
\hline Contacto directo de la piel & Corto plazo & Dermatitis, irritación y sensibilización \\
\hline Ingestión & Corto plazo & Toxicidad \\
\hline Inhalación & Corto plazo & Depresión del sistema nervioso central \\
\hline Inhalación & Mediano plazo & $\begin{array}{c}\text { Falta de coordinación, atención y deterioro del } \\
\text { equilibrio. }\end{array}$ \\
\hline Inhalación & Largo plazo & Daños hepáticos, renales o del sistema nervioso \\
central.
\end{tabular}

Elaboración propia a partir de [5].

Una forma de prevenir los efectos en la salud por uso y exposición a sustancias químicas es poner en práctica procesos de evaluación de riesgos, estos procesos utilizan métodos y técnicas aplicables a la evaluación de peligros, exposición y daños potenciales a la salud de los trabajadores. Según la Organización Mundial de la Salud (OMS), este proceso de se inicia con la formulación del problema e incluye los siguientes cuatros etapas fundamentales: 1) identificación del peligro, 2) caracterización del peligro, 3) evaluación de la exposición y 4) caracterización de riesgos. El riesgo depende de factores como: la cantidad utilizada de una sustancia química, el tiempo de exposición y el nivel toxicidad del producto químico [6]. 
Actualmente destacan dos evaluaciones de riesgo químico: el modelo británico COSHH Essentials, desarrollado por el Ejecutivo de Salud y Seguridad (HSE, por sus siglas en inglés); y el modelo francés del Instituto Nacional de Investigación en Seguridad (INRS). El modelo británico proporciona un diagnóstico inicial, comprende la etapa de estimación del riesgo (potencial) y no propiamente para determinar el nivel de riesgo existente [7]. El método del INRS es el más utilizado debido a que realiza una estimación más cercana a la realidad del riesgo laboral, considerando mayor cantidad de variables durante el proceso de evaluación, entre estas: propiedades de las sustancias químicas, cantidades utilizadas, forma de uso, valores límite umbrales y nivel de peligrosidad [8]. En el presente trabajo de investigación se determinó el nivel del riesgo químico por inhalación de sustancias químicas (NRQI) del proceso de impresión en una industria gráfica, para lo cual se identificaron y evaluaron los peligros y/o los riesgos químicos presentes en el ambiente laboral; mediante la metodología del INRS.

\section{Metodología}

Se realizó la evaluación del riesgo químico por inhalación (RQI) según las sustancias químicas utilizadas en una industria gráfica, durante un periodo 13 meses (entre mayo del 2018 y junio del 2019). La misma, comprendió las etapas de: a) identificación, b) valorización y c) priorización del RQI de las sustancias, utilizadas en el proceso productivo de la industria gráfica. Se utilizó la metodología de evaluación del riesgo químico denominada "método del INRS".

\section{Identificación del riesgo químico}

Mediante la realización de 15 visitas guiadas por funcionarios de la industria gráfica se estudió el diagrama de flujo general del proceso mediante el uso de balances de masa y energía. Durante las inspecciones de campo se ejecutaron: entrevistas, encuestas, listas de chequeo y reuniones con grupos focales de la industria en estudio. Se realizaron además reuniones con expertos internos (de la empresa) y externos en el tema de evaluación del riesgo químico, parte de las actividades del trabajo de campo incluyo la realización de talleres y sesiones de trabajo con funcionarios de la planta productiva. Producto de las actividades mencionadas anteriormente se generó una base de datos con los principales peligros químicos (PQs) y riesgos químicos (RQs) del proceso productivo de industria gráfica

Las listas de chequeo utilizadas evaluaron parámetros relativos a la gestión de productos químicos como: tipo de etiquetado según Sistema Globalmente Armonizado (GHS), uso de criterios de compatibilidad química para almacenamiento, condiciones de almacenamiento (ventilación, drenajes, señalización, iluminación y tipo de estantería), estado de los recipientes donde se almacenan, dispositivos de retención en caso de derrame o fuego, materiales de seguridad, equipo de protección personal, capacitación al personal en temas de seguridad e información sobre el seguimiento de la salud de los colaboradores.

Por su parte las encuestas higiénicas aplicadas a los funcionarios, permitió identificar y tabular información relacionada con el uso de sustancias químicas como: composición, cantidades utilizadas, frecuencia de uso, frases $\mathrm{R}$ y/o $\mathrm{H}$ y peligrosidad según NFPA (anexo 1 y 2). Los datos recopilados se complementaron y verificaron con fuentes de información como: hojas de datos de seguridad de los materiales (MSDS, por sus siglas en inglés) y bases de datos de instituciones internacionales como: La Administración de Seguridad y Salud de los Estados Unidos (OSHA, por sus siglas en inglés), la Agencia de Protección Ambiental de los Estados Unidos (EPA, por sus siglas en inglés), Instituto Nacional para la Seguridad y Salud Ocupacional de Estados Unidos (NIOSH, por sus siglas en inglés) y el Instituto Nacional de Seguridad e Higiene en el Trabajo de España. (INSHT). 


\section{Valorización del riesgo químico}

La cuantificación (valoración) del NRQI mediante la metodología del INRS consideró las siguientes variables: clase de peligro según frases de seguridad de las sustancias, cantidad de sustancia química utilizada, frecuencia de exposición a la sustancia química, la determinación de la exposición potencial, la volatilidad de las sustancias, el procedimiento de uso, formas de protección colectiva y los valores limites ambientales (VLA) [8]. En los siguientes apartados se describe la forma para la asignación de los valores de las variables según la metodología INRS.

\section{Clase de peligro $(P)$}

El valor para la clase de peligro se determina para cada sustancia química mediante las frases $\mathrm{R} \circ \mathrm{H}$, y/o los VLA en mg/m³, y/o el tipo de materiales y procesos. En función a estas variables una sustancia química puede obtener un valor para la clase de peligro 1, 2, 3, 4 y 5, siendo el valor 5, el que representa el mayor peligro hacia la salud

\section{Clase de cantidad (Q)}

El valor asignado a la clase de cantidad se obtuvo mediante las masas y/o volúmenes diarios de sustancia utilizada: (cuadro 2). Para las sustancias químicas sin información, se procedió con el cálculo del valor de la clase de cantidad mediante la aplicación del índice Qi/Qmax, esto es cantidad almacenada de sustancia de interés entre la cantidad de aquella sustancia almacenada en mayor volumen.

Cuadro 2. Clases de cantidad en función de la cantidad utilizada de la sustancia. Elaboración propia a partir de Aguilar et al [8].

\begin{tabular}{|c|c|c|}
\hline $\begin{array}{c}\text { Clase de cantidad para } \\
\text { sustancias con riesgo por } \\
\text { inhalación }\end{array}$ & Cantidad/día & Qi/Qmax \\
\hline 1 & $<100 \mathrm{~g} \mathrm{O} \mathrm{mL}$ & $<1 \%$ \\
\hline 2 & $\geq 100 \mathrm{~g} \mathrm{O} \mathrm{mL} \mathrm{y}<10 \mathrm{~kg} \circ \mathrm{L}$ & $\geq 1 \% \mathrm{a}<5 \%$ \\
\hline 3 & $\geq 10 \mathrm{~kg} \mathrm{O} \mathrm{L} \mathrm{y}<100 \mathrm{~kg} \mathrm{OL}$ & $\geq 5 \% \mathrm{a}<12 \%$ \\
\hline 4 & $\geq 100 \mathrm{~kg} \circ \mathrm{L} \mathrm{y}<1000 \mathrm{~kg} \circ \mathrm{L}$ & $\geq 12 \% \mathrm{a}<33 \%$ \\
\hline 5 & $\geq 1000 \mathrm{~kg} \circ \mathrm{L}$ & $\geq 33 \% \mathrm{a}<100 \%$ \\
\hline
\end{tabular}

Clase de frecuencia $(F)$

Los valores de la clase de frecuencia en el uso de las sustancias químicas se determinaron de acuerdo con lo mostrado en el cuadro 3.

Cuadro 3. Clases según frecuencia de uso de la sustancia química.

\begin{tabular}{|c|c|c|c|c|}
\hline Uso & Ocasional & Intermitente & Frecuente & Permanente \\
\hline Día & $\leq 30 \mathrm{~min}$ & $>30 \mathrm{~min} \mathrm{a} \leq 120 \mathrm{~min}$ & $>2 \mathrm{~h} \mathrm{a} \leq 6 \mathrm{~h}$ & $>6 \mathrm{~h}$ \\
\hline Semana & $\leq 2 \mathrm{~h}$ & $>2 \mathrm{~h}$ a $8 \mathrm{~h}$ & 1 día a 3 días & $>3$ días \\
\hline Mes & 1 día & 2 días a 6 días & 7 días a 15 días & $>15$ días \\
\hline Año & $\leq 15$ días & $>15$ días a $\leq 2$ meses & $>2$ meses a $\leq 5$ meses & $>5$ meses \\
\hline Clase & 1 & 2 & 3 & 4 \\
\hline
\end{tabular}

Elaboración propia a partir de Aguilar et al [8]. 


\section{Clase de exposición potencial (EP)}

Los valores de la clase de exposición potencial para cada uno de los productos químicos se determinaron en función a los valores obtenidos para las clases de cantidad y de frecuencia de uso, obteniéndose valores de 0, 1, 2, 3 y 4. El mayor valor (4) representa la mayor exposición potencial a determinado producto químico.

\section{Clase y puntuación del riesgo potencial (RP)}

Este valor se generó mediante la combinación de los valores asignados para las clases de peligro y las clases de exposición potencial. La combinación de estas dos variables permite obtener valores de riesgo potencial de 1, 2, 3, 4 y 5. El valor 5 representa el mayor riesgo potencial del producto químico evaluado.

El valor del riesgo potencial se obtiene de acuerdo con el valor de la clase de riesgo potencial, de forma que, si la clase es 1, 2, 3, 4 o 5; el valor del riesgo será 1, 10, 100, 1000 o 10000 , respectivamente.

\section{Clase y puntuación por volatilidad de las sustancias (V)}

Este valor se determinó con la temperatura de ebullición de la sustancia más volátil contenida en cada producto químico utilizado en la industria. La temperatura de ebullición se obtuvo de las MSDS. Mediante el gráfico de la figura 1 y considerando una temperatura de trabajo de 25 ${ }^{\circ} \mathrm{C}$, se obtuvo el dato numérico de la clase de volatilidad.

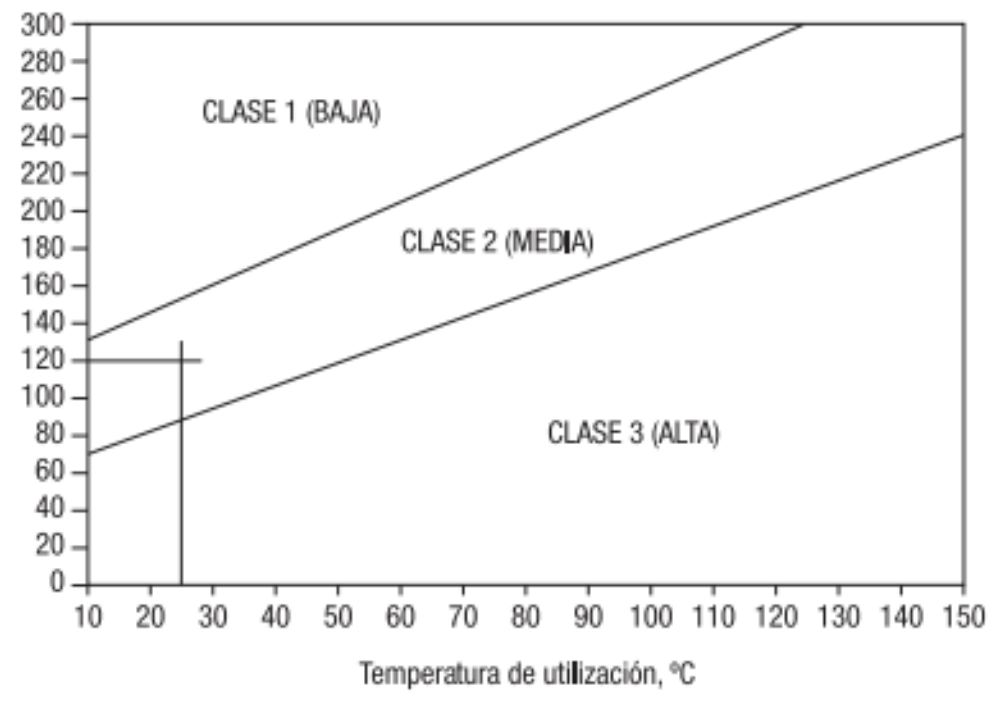

Figura 1. Tendencia por volatilidad de la sustancia [8].

\section{Clase y puntuación de procedimiento de uso (PU)}

El valor asignado a la clase de procedimiento de uso se basó en la descripción del procedimiento de uso de las sustancias químicas según el tipo proceso. Para un proceso de uso tipo dispersivo se asigna un valor de 4 (puntuación de 1), para un uso en tanques o conductos abiertos se asigna una clase de 3 (puntuación 0,5), si se utiliza en tanques cerrados con apertura ocasional se asigna un valor de 2 (puntuación 0,05 ) y si el uso es en tanques totalmente cerrados la clase es de 1 (puntuación 0,001). 
Clase y puntuación del tipo de protección colectiva en la industria (PC)

La puntuación de esta categoría se determinó utilizando la información del cuadro 4.

Cuadro 4. Determinación de puntuación, de acuerdo con las condiciones de trabajo.

\begin{tabular}{|c|c|c|}
\hline Tipo protección colectiva & Clase & Puntuación \\
\hline Espacio con aberturas limitadas de entrada y salida & 5 & 10 \\
\hline Espacio con ausencia de ventilación mecánica & 4 & 1 \\
\hline Trabajo a la intemperie, ventilación mecánica general & 3 & 0,7 \\
\hline $\begin{array}{c}\text { Campana superior, rendija de aspiración, mesa con aspiración. } \\
\text { Aspiración integrada a la herramienta }\end{array}$ & 2 & 0,1 \\
\hline Captación envolvente (campana de laboratorio) & 1 & 0,001 \\
\hline
\end{tabular}

Elaboración propia a partir de Aguilar et al [8].

\section{Puntuación según los valores límites ambientales (VLA)}

Los VLA ( $\left.\mathrm{mg} / \mathrm{m}^{3}\right)$ de cada sustancia se obtuvieron de la MSDS. El valor de la puntuación se asigna de la siguiente manera: valor de 1 para un VLA $>0,1$, valor 10 para $0,01<\mathrm{VLA} \leq 0,1$, valor 30 para un 0,001 $<$ VLA $\leq 0,01$ y valor de 100 para un VLA $\leq 0,001$

\section{Priorización del riesgo químico}

El valor final del RQI para cada sustancia química se obtuvo mediante la ecuación:

$$
P_{\text {inh }}=P_{\text {riesgo potencial }} x P_{\text {volatilidad }} x P_{\text {procedimiento }} x P_{\text {protección colectiva }} x F C_{V L A} \text { (Ecuación 1) }
$$

EI NRQI de cada sustancia química se priorizó de acuerdo con lo mostrado en el cuadro 5

Cuadro 5. Caracterización del riesgo por inhalación.

\begin{tabular}{|c|c|c|}
\hline $\begin{array}{c}\text { Puntuación del riesgo por } \\
\text { inhalación }\end{array}$ & $\begin{array}{c}\text { Prioridad de } \\
\text { acción }\end{array}$ & Caracterización del riesgo \\
\hline$>1000$ & 1 & Riesgo muy elevado (medidas correctoras inmediatas) \\
\hline$>100 \mathrm{a} \leq 1000$ & 2 & $\begin{array}{c}\text { Riesgo moderado. Necesita probablemente medidas } \\
\text { correctoras y/o una evaluación más detallada } \\
\text { (mediciones). }\end{array}$ \\
\hline$\leq 100$ & 3 & Riesgo bajo (sin necesidad de modificaciones). \\
\hline
\end{tabular}

Elaboración propia a partir de Aguilar et al [8].

\section{Discusión y resultados}

\section{Identificación de riesgos químicos}

Las visitas de campo realizada en la industria, permitió determinar las sustancias químicas utilizadas en el proceso productivo, así como cantidades y frecuencias de uso. Los principales productos químicos utilizados son: limpiadores con distintas composiciones (ver anexo 1), isopropanol, thinner, tintas, aceite, goma protectora y revelador de planchas, los cuales se consumen en cantidades entre los 0,5-77,0 litros mensuales. 
Por otra parte, el análisis integral de los datos obtenidos con las diferentes encuestas y lista de chequeo, así como la consulta de otras fuentes de información mencionadas anteriormente, permitió identificar los principales peligros, características fisicoquímicas y toxicológicas de los productos químicos. Esta información se encuentra tabulada en los anexos 1 y 2, información fundamental para establecer el NRQI actual de la industria evaluada. Los datos incluidos en los anexos 1 y 2 se resume en el cuadro 6.

Cuadro 6. Síntesis de la situación riesgo químico actual en la industria gráfica.

\begin{tabular}{|c|c|}
\hline \multicolumn{2}{|c|}{$\begin{array}{l}\text { Aspecto: Uso de sustancias químicas con alto contenido de VOCs, compuestos cancerígenos y con } \\
\text { alta toxicidad }\end{array}$} \\
\hline Síntesis de la situación de riesgo & Indicadores \\
\hline $\begin{array}{l}\text { Fuentes de generación: uso de compuestos químicos como materias primas para } \\
\qquad \text { la impresión de documentos. }\end{array}$ & \multirow{10}{*}{$\begin{array}{c}\text { En total se } \\
\text { estima que se } \\
\text { utilizan } 87 \mathrm{~L} / \mathrm{mes} \\
\text { de sustancias } \\
\text { químicas (ver } \\
\text { anexo 1). }\end{array}$} \\
\hline $\begin{array}{l}\text { Información de las sustancias: Las sustancias químicas utilizadas contienen las } \\
\text { siguientes características: }\end{array}$ & \\
\hline -El 75\% de las sustancias químicas son de uso diario. & \\
\hline $\begin{array}{l}\text {-El } 12,5 \% \text { de las sustancias presentan valores de } 3 \text { y } 4 \text { en salud en la escala } \\
\text { de la Asociación Nacional de Protección contra el Fuego de los Estados Unidos } \\
\text { (NFPA, por sus siglas en ingles), indicativo de su alta toxicidad. }\end{array}$ & \\
\hline $\begin{array}{l}\text {-El 62,5\% presentan una temperatura de inflamación entre un rango de } 12^{\circ} \mathrm{C} \text { a } \\
\qquad 62^{\circ} \mathrm{C} \text {, indicativo de su alta peligrosidad por incendio. }\end{array}$ & \\
\hline $\begin{array}{c}\text {-Un } 56,3 \% \text { de las sustancias son consideradas mortales por inhalación ya } \\
\text { que presentan valores de categoría 1, según escala del Sistema Globalmente } \\
\text { Armonizado (SGA). }\end{array}$ & \\
\hline -Un $12,5 \%$ son clasificadas como mortales en caso de ingestión & \\
\hline -El 6,25\% son clasificadas como mortales por contacto con la piel. & \\
\hline $\begin{array}{c}\text {-De las sustancias químicas } 3 \text { son clasificadas como mutagénicas y } 7 \text { como } \\
\text { cancerígenas. }\end{array}$ & \\
\hline $\begin{array}{c}\text { Disposición de residuos: los residuos químicos generados son tratados por } \\
\text { incineración por un gestor externo. }\end{array}$ & \\
\hline
\end{tabular}

Respecto a la gestión de productos y residuos químicos (producto de las visitas de campo y aplicación de la lista de chequeo) se identificaron 9 aspectos positivos y 16 aspectos negativos. Uno de los principales aspectos positivos es que la industria mantiene un inventario actualizado de productos químicos y un buen sistema de compras (no se encontró productos expirados).

De los aspectos negativos se evidenció la colocación de materiales en la parte superior de los gabinetes exclusivos para productos químicos. Esto además de ser una práctica de trabajo inadecuada, implica un mayor riesgo, debido a que los gabinetes no disponen de protección contra incendios, además de la inexistencia de protección en los bordes de los gabinetes para recolección derrames.

\section{Valoración del riesgo químico por inhalación}

Una vez recolectada la información preliminar, se procedió a la asignación de valores a los factores incluidos en la metodología del INRS, según cada sustancia química utilizada en la industria gráfica. A continuación, se detalla la forma de asignación de los valores:

- $P$ : se asignó en función a las frases $\mathrm{R}$ y $\mathrm{H}$ de las MSDS de los productos químicos, disponibles en el registro digital de la industria.

- Q: se estableció considerando la cantidad de producto químico utilizado mensualmente según inventario de la industria (anexo 1). 
- F: se estableció según la frecuencia de uso del producto químico, determinado a través de las encuestas higiénicas al personal de producción de impresiones de la industria gráfica (anexo 1).

- EP: lo constituye la relación entre los valores de las clases de frecuencia y cantidad.

- RP: es la relación entre los valores de las clases de peligro y de exposición potencial.,

- V: Valor obtenido según la relación (figura 1) entre la temperatura de ebullición de la sustancia (anexo 1) y la temperatura de trabajo $\left(25^{\circ} \mathrm{C}\right)$ en las instalaciones de la industria.

- PU: Para un 40,0 \% de los productos químicos el valor asignando fue de 1 (procedimiento de uso dispersivo) y para un 40,0 \% el valor fue de 0,5 (procedimiento de uso abierto) Para productos específicos como la goma y el revelador se asignó un valor de 0,05 (procedimiento de uso cerrado), debido a que estos productos se utilizan en tanques cerrados. Los cartuchos de tinta permanecen cerrados completamente y por esta razón se le asignó un valor de 0,001.

- PC: Para un total de 12 productos químicos se asignó un valor de 10, debido a que estos productos son manipulados en zonas con ausencia de circulación de aire (no se dispone de sistemas mecánicos y/o naturales de inyección y extracción de aire). Para los productos químicos manipulados en preimpresión, los cuales son un total de 3, se les incluyó un valor de 1 , debido que estos productos son manipulados en áreas del taller que disponen de apertura de puertas durante el día.

- VLA: se colocó un valor de 1 a todos los productos químicos, debido a que todos presentan un VLA > 1 (anexo 2).

En el cuadro 7 se detallan los productos químicos y los valores de las variables evaluadas, así como el valor final del RQI para cada producto (ecuación 1). Del total de 15 productos químicos, un 53,3\% presentan un NRQI clasificado como muy elevado, lo que implica la ejecución de medidas correctoras inmediatas. Entre estos productos químicos se destacan principalmente el thinner y el limpiador de rodillos, cuya composición se observa en el anexo 1

Priorización del nivel de riesgo químico por inhalación

La figura 2 muestra la caracterización final del NRQI para cada sustancia química utilizada en el proceso de impresión y, por ende, su priorización para futuras acciones preventivas y correctivas, tanto desde el punto de vista operativo como de gestión administrativa.

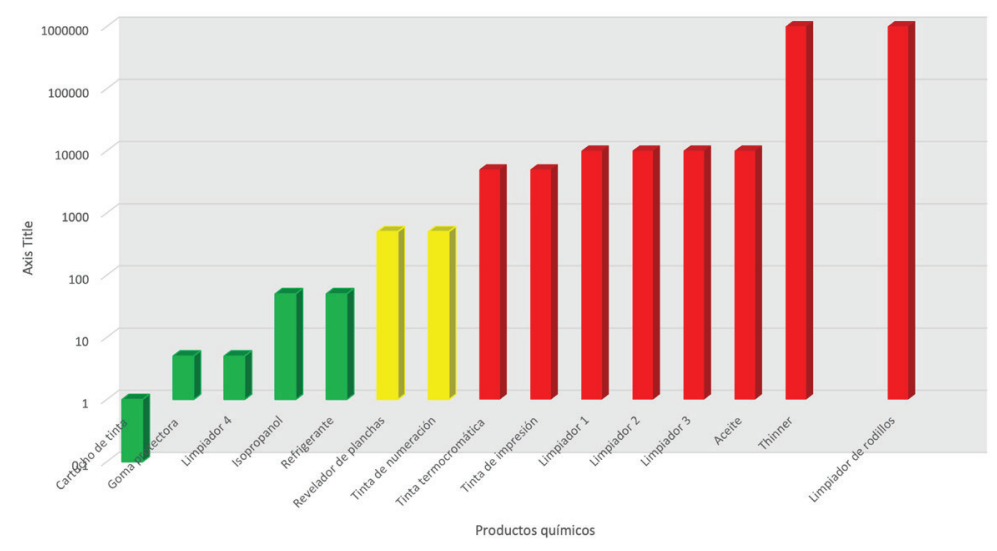

Figura 2. Priorización de sustancias según su riesgo por inhalación. 
Cuadro 7. Evaluación del riesgo químico en los diferentes subprocesos.

\begin{tabular}{|c|c|c|c|c|c|c|c|c|c|c|c|c|}
\hline \multirow{3}{*}{$\begin{array}{l}\text { Proceso } \\
\text { Sustancia }\end{array}$} & \multicolumn{12}{|c|}{ Preimpresión } \\
\hline & \multicolumn{5}{|c|}{ Clase de: } & \multicolumn{7}{|c|}{ Puntuación de: } \\
\hline & Peligro & Cantid & Frecuen & $\begin{array}{c}\text { Exposición } \\
\text { potencial }\end{array}$ & $\begin{array}{l}\text { Riesgo } \\
\text { potencial }\end{array}$ & $\begin{array}{l}\text { Riesgo } \\
\text { potencial }\end{array}$ & Volatilid & $\begin{array}{l}\text { Procedim } \\
\text { de uso }\end{array}$ & $\begin{array}{l}\text { Protección } \\
\text { colectiva }\end{array}$ & VLA & $\begin{array}{l}\text { Total } \\
\text { riesgo }\end{array}$ & $\begin{array}{l}\text { Clasific del } \\
\text { riesgo }\end{array}$ \\
\hline $\begin{array}{c}\text { Revelador de } \\
\text { planchas }\end{array}$ & 4 & 2 & 4 & 2 & 3 & 100 & 100 & 0,05 & 1 & 1 & 500 & Moderado \\
\hline Cartucho de tinta & 4 & 2 & 3 & 2 & 3 & 100 & 1 & 0,001 & 1 & 1 & 0,1 & Bajo \\
\hline $\begin{array}{c}\text { Goma protectora } \\
\text { de planchas }\end{array}$ & 4 & 1 & 2 & 1 & 3 & 100 & 1 & 0,05 & 1 & 1 & 5 & Bajo \\
\hline Proceso & \multicolumn{12}{|c|}{ Impresión } \\
\hline Limpiador 1 & 3 & 2 & 2 & 2 & 2 & 10 & 100 & 1 & 10 & 1 & 10000 & Alto \\
\hline Limpiador 2 & 3 & 2 & 2 & 2 & 2 & 10 & 100 & 1 & 10 & 1 & 10000 & Alto \\
\hline Thinner & 5 & 2 & 3 & 2 & 4 & 1000 & 100 & 1 & 10 & 1 & 1000000 & Alto \\
\hline Isopropanol & 2 & 2 & 4 & 2 & 1 & 1 & 10 & 0,5 & 10 & 1 & 50 & Bajo \\
\hline Limpiador 3 & 3 & 2 & 2 & 2 & 2 & 10 & 100 & 1 & 10 & 1 & 10000 & Alto \\
\hline $\begin{array}{l}\text { Limpiador de } \\
\text { rodillos }\end{array}$ & 5 & 1 & 2 & 1 & 4 & 1000 & 100 & 1 & 10 & 1 & 1000000 & Alto \\
\hline $\begin{array}{c}\text { Tinta de } \\
\text { numeración }\end{array}$ & 4 & 2 & 4 & 2 & 3 & 100 & 1 & 0,5 & 10 & 1 & 500 & Moderado \\
\hline Limpiador 4 & 2 & 2 & 3 & 2 & 1 & 1 & 1 & 0,5 & 10 & 1 & 5 & Bajo \\
\hline Aceite & 3 & 2 & 2 & 2 & 2 & 10 & 100 & 1 & 10 & 1 & 10000 & Alto \\
\hline Refrigerante & 3 & 2 & 4 & 2 & 2 & 10 & 1 & 0,5 & 10 & 1 & 50 & Bajo \\
\hline $\begin{array}{c}\text { Tinta } \\
\text { termocromática }\end{array}$ & 3 & 2 & 4 & 2 & 2 & 10 & 100 & 0,5 & 10 & 1 & 5000 & Alto \\
\hline Tinta de impresión & 3 & 2 & 4 & 2 & 2 & 10 & 100 & 0,5 & 10 & 1 & 5000 & Alto \\
\hline
\end{tabular}

*Información detallada de las sustancias mostradas en anexo 1 y 2. 
Los productos químicos con los menores (color verde) valores de NRQI son: la tinta en cartucho $(0,1)$, el refrigerante (50), el limpiador 4 (5), el isopropanol (50) y la goma protectora (5). Estos productos son utilizados principalmente; en la impresión, para mantener la temperatura de la máquina de impresión, como limpiador, como disolución de fuente y para la protección de placas. Las cantidades utilizadas son de entre $2 \mathrm{~L} / \mathrm{mes}$ y $40 \mathrm{~L} / \mathrm{mes}$, y con una frecuencia de uso no mayor a 2 veces por semana. Los menores valores del NRQI para estos productos químicos se deben a los puntos de ebullición, los cuales son mayores a los $100{ }^{\circ} \mathrm{C}$, por lo tanto, el valor de la clase de volatilidad es bajo (1 a 10). El resto de los productos (Por ejemplo: el thinner, revelador, limpiador de rodillos, entre otros) presentan valores para la clase de volatilidad de 100, por lo tanto, reportan valores mayores de NRQI. Otra variable importante que influye en el valor del riesgo final es el procedimiento de uso, todos estos productos químicos con bajos NRQI son utilizados en contenedores cerrados o que se pueden abrir de forma ocasional, por lo tanto, su valor varía entre 0,001 y 0,05.

Contrariamente, los productos con mayores (color rojo) NRQI son el thinner y el limpiador de rodillos, los cuales son utilizados en el área de impresión para la limpieza de las máquinas, con una frecuencia de uso continua, se utilizan cantidades entre los 0,7 L/mes y los 20,0 L/mes. Los valores obtenidos de RQI (1000000) se debe principalmente a la variable denominada la clase de peligro, con las frases H340 y 350 para el thinner y la frase R45 para el limpiador. La frase R45 indica que es una sustancia que puede "causar cáncer" [9]. La MSDS del limpiador indica "Este producto contiene los siguientes productos químicos conocidos por el estado de California como causantes de cáncer: el benceno y de daños reproductivos: benceno, tolueno". Por otra parte, las frases H340 y H350 señalan: pueden causar efectos genéticos y cáncer [9], respectivamente. El thinner contiene tolueno, los valores de campo obtenidos para el tolueno en la industria gráfica evaluada están entre los rangos de 73,3 ppm a 123,0 ppm, estas concentraciones son superiores al VLA (50,0 ppm, INSHT)

En general, los limpiadores 1, 2 y 3 utilizados contienen destilados de petróleo, alcoholes, cetonas, alcanos, cicloalcanos y aromáticos; los cuales presentan RQI alto para la exposición por vía inhalatoria, Sin embargo, el limpiador 4 presenta un NRQI bajo, debido a la baja volatilidad y clase de peligro, siendo una buena opción para sustituir otros disolventes utilizados durante la limpieza.

El revelador y la tinta de numeración muestran valores intermedios (color amarillo) para el NRQI. El revelador se utiliza en preimpresión, para la elaboración diaria de las planchas en cantidades de $60 \mathrm{~L} / \mathrm{mes}$. El valor intermedio de NRQI asignado para este producto se debe principalmente al procedimiento de uso en tanque cerrado $(0,05)$, sin embargo, el valor del riesgo obtenido de las frases $\mathrm{R} / \mathrm{H}$ es alto (4). En el caso de la tinta de numeración, esta se utiliza continuamente y en una cantidad de $16 \mathrm{~L} / \mathrm{mes}$; además presenta una puntuación de volatilidad baja, ya que su temperatura de ebullición es superior a los $200^{\circ} \mathrm{C}$.

Los resultados obtenidos indican que el nivel de NRQI para la industria gráfica es elevado y por tanto se deben implementar medidas para su disminución. Las acciones deben estar dirigidas a: 1) regular la forma de uso y manejo de sustancias químicas, 2) substituir productos químicos utilizados actualmente y 3) seguimiento de la salud de los funcionarios.

La primera propuesta comprende desarrollar y poner en práctica un "Protocolo para el Manejo de Productos Químicos", el cual debe contener y evaluar: 1) criterios de compra, 2) forma de almacenamiento, 3) buenas prácticas de trabajo (evitar excesos y desperdicios de materia prima, disminuir la generación de desechos, 4) uso de equipo de protección personal (EPP) y 5) adecuada gestión de desechos $[10,11]$. La puesta en marcha de esta propuesta implica procesos de capacitación y fiscalización. 
La segunda acción tiene como objetivo principal la eliminación y sustitución de los productos actualmente utilizados que contienen: porcentajes superiores al 10\% en VOCs (incluye productos con temperaturas de inflamación menores a los $60^{\circ} \mathrm{C}$ ), componentes clasificados como cancerígenos y altas toxicidades (valor 3 y 4 en escala de la NFPA).

Finalmente, la tercera propuesta se enfoca a la vigilancia a la salud de los trabajadores, mediante exámenes médicos en orina y sangre, realización de pruebas físicas, así como la realización de entrevistas al personal expuesto a productos químicos para un mejor control y seguimiento de sus estados de salud.

Los funcionarios del sitio estudiado pueden estar expuestos a altas concentraciones de vapores orgánicos (VOCs), en especial al tolueno proveniente del thinner; razón por la cual se sugiere realizar exámenes médicos en orina y sangre. En el caso del tolueno se puede cuantificar la cantidad de o-cresol o ácido hipúrico en orina [12]. Exposiciones repetidas de tolueno puede afectar el sistema nervioso central provocando dolores de cabeza, incoordinación, perdidas de visión y audición. Algunos efectos adversos a largo plazo pueden afectar el hígado, los riñones, los pulmones y el sistema inmunitario [12]. Por lo anterior, la vigilancia a la salud de los trabajadores es importante para medir posibles afectaciones por exposición ocupacional y tomar decisiones en pro de la salud de los funcionarios.

\section{Conclusiones}

Las visitas de campo a la empresa, la aplicación encuestas higiénicas, listas de chequeo y la consulta de otras fuentes de información, permitieron conocer el flujo del proceso de impresión, las sustancias utilizadas, así como la cantidad y frecuencia de uso y la identificación de los principales riesgos y peligros en la industria gráfica.

Lo anterior dio como resultado la sistematización de la información fisicoquímica y toxicológica de las 15 sustancias utilizadas por la industria gráfica. Con respecto a los valores fisicoquímicos, se encontró que el 12,5\% presentaron valores en NFPA de 3 y 4 en salud y el 62,5\% presentan una temperatura de inflamación entre un rango de $12{ }^{\circ} \mathrm{C}$ y $62^{\circ} \mathrm{C}$, aumentando esto, el riesgo por inhalación. Las propiedades toxicológicas indicaron que un 56,3\% de las sustancias son consideradas mortales por inhalación, un 12,5\% clasificadas como mortales en caso de ingestión y un 6,25 \% como mortales por contacto con la piel. Del total de las sustancias químicas 3 fueron clasificadas como mutagénicas y 7 como cancerígenas.

En la evaluación del NRQI con la metodología del INRS, se determinó que, del total de 15 productos químicos, el 53,3 \% presentan un RQI muy elevado. Los productos que presentaron el mayor valor de RQI (1000000) son; el thinner y el limpiador de rodillos. Por el contrario, se identificaron cinco productos con valores bajos de riesgo: la tinta en cartucho $(0,1)$, el refrigerante (50), el limpiador 4 (5), el isopropanol (50) y la goma protectora (5).

Se determinó que la industria gráfica en estudio presenta un RQI alto, por lo que es necesario desarrollar y poner en práctica medidas para su disminución. Se proponen 3 tipos de acciones dirigidas a 1) crear disposiciones para el manejo seguro y adecuado de sustancias químicas, 2) sustitución de productos con alto contenido de VOCs, temperaturas de inflamación bajas y con alta toxicidad, y 3) vigilancia a la salud de los trabajadores expuestos.

\section{Referencias}

[1] Medaglia, C., and Vargas, F. (2013). Oportunidades para la industria gráfica costarricense en Panamá [online]. PROCOMER, San Jose, Costa Rica. Disponible: https://procomer.com/uploads/downloads/43aa5e0839d 8cf5d51efa444ec45e7064184b7fa.pdf 
[2] Occupational Safety and Health Branch Labour Department. (2004). Chemical Safety in the Workplace: Guidance Notes on Chemical Safety in Printing Industry. Disponible: https://www.labour.gov.hk/eng/public/ os/C/GN_Printing.pdf

[3] Knezović, Z., Trgo, M., Sutlović, D. (2016). Monitoring mercury environment pollution through bioaccumulation in meconium, Process Saf. Environ, 101, 2-8. DOI: 10.1016/j.psep.2016.01.013

[4] Prica, M. Kecie, V. Adamovié, S. Radonié, J. Turk, M. "Occupational Exposure to Hazardous Substances in Printing Industry," Proceedings of 8th International Engineering Symposium, Bánki [online], 1-95, 2016.

[5] Ministerio de Trabajo y Asuntos Sociales. Ministerio de Medio Ambiente. (2011). Manual de Buenas Prácticas Ambientales en la Familia Profesional: Industrias Gráficas; España. Disponible: https://www.miteco.gob.es/es/ calidad-y-evaluacion-ambiental/temas/red-de-autoridades-ambientales-raa-/indgrafica_tcm30-166749.pdf

[6] Organización Mundial de la Salud. Programa Internacional sobre Seguridad de las Sustancias Químicas (IPCS). (2010). Herramienta de Evaluación de Riesgos para la Salud Humana de la OMS: Peligros Químicos. Disponible: https://www.who.int/ipcs/publications/ra_toolkit/es/

[7] Marín, D. Montes de Oca, O. González, J. (2017). Evaluación de riesgos químicos en un laboratorio de Química Analítica por el método COSSH Essentials [online], Ciencia en su PC, 91-106. Disponible: https:// www.redalyc.org/pdf/1813/181353026008.pdf

[8] Aguilar, J. Bernaola, M. Gálvez, V. (2017). Riesgo Químico: Sistemática para la Evaluación Higiénica. Instituto de Seguridad e Higiene en el Trabajo de España. Disponible: https://www.insst.es/documents/94886/96076/Rie sgo+qu\%C3\%ADmico+Sistem\%C3\%A1tica+para+la+evaluaci\%C3\%B3n+higi\%C3\%A9nica.pdf/55fdf7ce$7 f 1 b-43 b 4-97 d 2-3 b 36 b 4574 c 9 e$

[9] Guardino, X. (2015). Regulación UE sobre productos químicos (II). Reglamento CLP: aspectos básicos. Disponible: https://www.insst.es/

[10] García, C., Leone, I. D., \& Williams, E. (2014). Buenas Prácticas Ambientales en Industrias Gráficas y Afines [online]; Union Gráfica Argentina Regional, Rosario, Argentina, 11-31. Disponible pdf: https://www.rosario. gob.ar/web/sites/default/files/manual_graficos

[11] Avalo, R. (2014). Estrategia de Manejo Ambiental para una Industria Gráfica. Tesis para optar por el título de Ingeniero Ambiental. Universidad Nacional Agraria, Perú. Disponible pdf: https://www.academia. edu/36558486/Estrategia de Manejo Ambiental para una Industria Gr\%C3\%A1fica

[12] Instituto Nacional de Seguridad e Higiene en el Trabajo (INSST). 2007. Documentación Toxicológica para el Establecimiento del Límite de Exposición Profesional del Tolueno. 
Anexo 1. Propiedades físicas de los productos químicos utilizados en la industria gráfica.

\begin{tabular}{|c|c|c|c|c|c|c|c|c|c|c|c|c|c|}
\hline \multirow{2}{*}{$\begin{array}{c}\text { Subproceso } \\
\text { Producto } \\
\end{array}$} & \multicolumn{4}{|c|}{ Preimpresión } & \multicolumn{3}{|c|}{ NFPA } & \multirow{2}{*}{$\mathrm{pH}$} & \multirow{2}{*}{${ }^{\mathrm{T}} \mathrm{C} \mathrm{Cb}$} & \multirow{2}{*}{$\begin{array}{l}\text { T.Inflam } \\
{ }^{\circ} \mathrm{C}\end{array}$} & \multirow{2}{*}{$\begin{array}{l}\text { Presión de } \\
\text { vapor } \mathrm{kPa}^{*}\end{array}$} & \multicolumn{2}{|c|}{ Límites de inflamabilidad } \\
\hline & Composición & Cantid & Frec de uso & Frases $H / R$ & s & 1 & $\mathrm{R}$ & & & & & LIE & LSE \\
\hline Revelador & $\begin{array}{l}\text { 5\% Dietanolamina, } 10 \% \text { 2-fenoxietanol, 5\% } \\
\text { destilados de petróleo }\end{array}$ & $40 \mathrm{~L} / \mathrm{mes}$ & Diario & $\begin{array}{l}\mathrm{H} 302, \mathrm{H} 315, \mathrm{H} 318 \\
\mathrm{H} 373, \mathrm{H} 412\end{array}$ & 3 & 1 & 0 & 10,5 & 245 & 127 & 0,0013 & $1,4 \%$ & $9,0 \%$ \\
\hline $\begin{array}{l}\text { Goma protectora de } \\
\text { planchas }\end{array}$ & $\begin{array}{l}\text { 1\% Ácido aminotrimetilenfosfónico, 0,1\% } \\
\text { 5-Cloro-2-metil-2H-isotiazol-3-ona, 0,1\% } \\
\text { 2-Metil-2H-isotiazol-3-ona }\end{array}$ & $0,9 \mathrm{~L} / \mathrm{mes}$ & Diario & $\begin{array}{l}\text { R36/38, R23/24/25, } \\
\text { R34, R43, R50/53 }\end{array}$ & 1 & 0 & 0 & 2,9 & $>100$ & NR & 2,3 & NR & NR \\
\hline $\begin{array}{l}\text { Cartucho de tinta en } \\
\text { polvo }\end{array}$ & $\begin{array}{l}\text { 10\% carbón negro, } 1 \% \text { dióxido de titanio, } 70 \% \\
\text { Resina de poliéster, } 20 \% \text { polvo de ferrita, } 10 \% \\
\text { pigmento. }\end{array}$ & $\begin{array}{c}10 \\
\text { cartuchos / } \\
\text { mes }\end{array}$ & Diario & $\begin{array}{l}\mathrm{H} 226, \mathrm{H} 315, \mathrm{H} 332 \\
\text { H351 }\end{array}$ & 0 & 1 & 0 & NR & 145 & 31 & 5 & 1,1 & 6,1 \\
\hline Subproceso & \multicolumn{4}{|c|}{ Impresión } & \multicolumn{3}{|c|}{ NFPA } & \multirow{2}{*}{$\mathrm{pH}$} & \multirow{2}{*}{$\begin{array}{l}\text { T.Eb } \\
{ }^{\circ} \mathrm{C}\end{array}$} & \multirow{2}{*}{$\begin{array}{l}\text { T.Inflam } \\
{ }^{\circ} \mathrm{C}\end{array}$} & \multirow{2}{*}{$\begin{array}{l}\text { Presión de } \\
\text { vapor } \mathrm{kPa}\end{array}$} & \multicolumn{2}{|c|}{ Límites de inflamabilidad } \\
\hline Producto & Composición & Cantidad & Frec de uso & Frases H/R & $\mathrm{s}$ & 1 & $R$ & & & & & LIE & LSE \\
\hline Limpiador 1 & $\begin{array}{c}10 \% \text { ácido ortofosforico, } 10 \% \text { de destilados } \\
\text { de petróleo }\end{array}$ & $0,52 \mathrm{~L} / \mathrm{mes}$ & $\begin{array}{l}1-2 \text { veces por } \\
\text { semana }\end{array}$ & $\begin{array}{c}\text { R52/53, H319, } \\
\text { H412, R34, R65, } \\
\text { R10, R37, R51/53 }\end{array}$ & 3 & 2 & 0 & 2 & 158 & 62 & 0,016 & 0,5 & 7,0 \\
\hline Limpiador 2 & $\begin{array}{c}60 \% \text { destilados de petróleo y keroseno, } 10 \% \\
\text { ácido fosfórico, } 5 \% \text { de trimetilbenceno, } 5 \% \\
\text { xileno, } 5 \% \text { mestileno, }\end{array}$ & $6 \mathrm{~L} / \mathrm{mes}$ & $\begin{array}{l}1-2 \text { veces por } \\
\text { semana }\end{array}$ & $\begin{array}{l}\mathrm{H} 226, \mathrm{H} 315, \mathrm{H} 319 \\
\mathrm{H} 373, \mathrm{H} 336\end{array}$ & 2 & 3 & 0 & 2 & 150 & 42 & 0,4 & 0,7 & 5,0 \\
\hline Thinner & $\begin{array}{l}60 \% \text { aromáticos, alcohol metilico, cetonas, } \\
\text { hexanos, alcoholes, xileno, esteres. }\end{array}$ & $20,5 \mathrm{~L} / \mathrm{mes}$ & $\begin{array}{l}1-2 \text { veces por } \\
\text { semana }\end{array}$ & $\begin{array}{l}\mathrm{H} 225, \mathrm{H} 340, \mathrm{H} 350 \\
\mathrm{H} 373, \mathrm{H} 304\end{array}$ & 2 & 3 & 0 & NA & 98 & 43 & 0,3 & 1,0 & 13,5 \\
\hline Isopropanol & 99\% Isopropanol & $77 \mathrm{~L} / \mathrm{mes}$ & Diario & R11, R36, R67 & 2 & 3 & 0 & NA & 82 & 12 & 5,8 & 2,0 & 12,7 \\
\hline Limpiador 3 & $\begin{array}{c}60 \% \text { alcanos, } 10 \% \text { a } 30 \% \text { acetona, } 10 \% \text { a } 30 \\
\% \text { tolueno, } 10 \% \text { a } 30 \% \text { propanol, } 5 \% \text { a } 10 \% \\
\text { cicloalcanos }\end{array}$ & $17,5 \mathrm{~L} / \mathrm{mes}$ & $\begin{array}{l}1-2 \text { veces por } \\
\text { semana }\end{array}$ & $\begin{array}{l}\mathrm{H} 315, \mathrm{H} 319, \mathrm{H} 361 \mathrm{~d}, \\
\mathrm{H} 336, \mathrm{H} 373, \mathrm{H} 304\end{array}$ & 2 & 2 & 0 & NA & 114 & 15 & 4,9 & NR & NR \\
\hline Limpiador de rodillos & 99\% Destilados de petróleo & $0,7 \mathrm{~L} /$ mes & Diario & R45 & 2 & 3 & 0 & NA & 115 & 10 & 6,9 & 0,5 & 7,0 \\
\hline Limpiador 4 & $90 \%$ 2-(2-etoxietoxi) etil acetato & $4 \mathrm{~L} / \mathrm{mes}$ & Diario & $\mathrm{R} 19, \mathrm{R} 36 / 37 / 38$ & 2 & 0 & 1 & NA & 217 & 100 & 0,13 & 1,5 & $\mathrm{NR}$ \\
\hline Tinta de numeración & $\begin{array}{c}\text { 40\% Triacrilato de trimetilpropano, } \\
30 \% \text { diacrilato de } 1,6 \text {-hexanodiol, } 10 \% \\
\text { caprolactama de vinil, } 5 \% \text { alquilfenona, 0,2\% } \\
\text { éster acrilato } \\
\end{array}$ & $16 \mathrm{~L} / \mathrm{mes}$ & Diario & $\mathrm{R} 36 / 38, \mathrm{R} 43, \mathrm{R} 52 / 53$ & 1 & 0 & 0 & NA & $>200$ & $>60$ & $<0,0101$ & 2,0 & 11,8 \\
\hline Aceite & $97 \%$ aceite nafténico pesado & $4 \mathrm{~L} / \mathrm{mes}$ & Diario & $\mathrm{H} 336, \mathrm{H} 304$ & & & & NA & $>260$ & $>150$ & NR & NR & NR \\
\hline Refrigerante & $70 \%$ etilenglicol, $30 \%$ dietilenglicol & $21 \mathrm{~L} \mathrm{l} / \mathrm{mes}$ & Diario & H302, H361, H373 & 2 & 1 & 0 & 10,8 & 107 & NR & NR & NR & NR \\
\hline Tinta termocromática & Pigmento, $20 \%$ a 30\% destilados de petróleo & $10 \mathrm{~kg} / \mathrm{mes}$ & Diario & R65, R66 & 2 & 1 & 0 & NA & 115 & 10 & 6,9 & 0,5 & 7,0 \\
\hline Tinta impresión & $\begin{array}{c}\text { Pigmento, } 30 \% \text { destilados de petróleo, terbutil } \\
\text { hidroquinona menor a } 1 \% \text {, cobalto menor a } \\
1 \% \text {, manganeso menor a } 1 \%\end{array}$ & $\begin{array}{l}25 \mathrm{~L} / \text { mes } \\
\text { amarillo y } \\
\text { magenta. } \\
20 \mathrm{~L} / \text { mes } \\
\text { negro }\end{array}$ & Diario & $\begin{array}{l}\text { H302, H315, H317, } \\
\text { H319, H361f } \\
\text { H361d, H373, }\end{array}$ & 2 & 1 & 0 & NA & 115 & 10 & 6,9 & 0,5 & 7,0 \\
\hline
\end{tabular}

Fuente: Hojas de seguridad de las sustancias, proporcionadas por la industria gráfica, nombre y marca de las sustancias no colocadas por protección de imagen. 
Anexo 2. Propiedades toxicológicas de los productos químicos utilizados en la industria gráfica.

\begin{tabular}{|c|c|c|c|c|c|c|c|c|c|c|c|c|c|c|c|}
\hline \multirow[b]{2}{*}{ Producto } & \multicolumn{2}{|c|}{ LD50 (mg/kg) } & \multirow[b]{2}{*}{$\begin{array}{l}\text { LC50 } \\
(\mathrm{mg} / \mathrm{L})\end{array}$} & \multicolumn{3}{|c|}{ SGA } & \multicolumn{2}{|c|}{ Mutagénic } & \multicolumn{2}{|c|}{ Cancernogénico } & \multicolumn{5}{|c|}{ Valores TLV (ppm) } \\
\hline & $\begin{array}{l}\text { Toxicidad } \\
\text { oral aguda }\end{array}$ & $\begin{array}{c}\text { Toxicidad } \\
\text { dérmica }\end{array}$ & & Oral & Dérmica & Inhalación & $\mathrm{Si}$ & No & $\mathrm{Si}$ & No & TWA & IST & STEL & IST & Celling \\
\hline Revelador & 1,902 & 2,2014 & $>100$ & 4 & 4 & 2 & & $x$ & $x$ & & 2 & INSHT & NR & - & NR \\
\hline Goma protectora & 2,100 & $>6,310$ & 0,16 & 4 & 5 & 1 & & $x$ & & $x$ & 10 & ACGIH & NR & - & NR \\
\hline $\begin{array}{l}\text { Cartucho de tinta en } \\
\text { polvo }\end{array}$ & $>5000$ & $>5000$ & $>5000$ & 5 & 5 & 4 & & $x$ & & $x$ & 10 & NR & NR & - & NR \\
\hline Limpiador 1 & 1,53 & 2470 & NR & 1 & 2 & 1 & & $x$ & & $x$ & 1 & HSE & 2 & HSE & NR \\
\hline Limpiador 2 & $>5000$ & $>2000$ & $>5$ & 5 & 2 & 1 & & $x$ & $x$ & & 200 & OSHA & 1600 & ACGIH & NR \\
\hline Thinner & 1515 & 3333 & 16,42 & 3 & 2 & 1 & & $x$ & $x$ & & 100 & ACGIH & NR & - & NR \\
\hline Isopropanol & 5045 & 5045 & 16000 & 5 & 5 & 3 & & $x$ & & $x$ & 400 & OSHA & 500 & OSHA & NR \\
\hline Limpiador 3 & NR & NR & NR & 1 & 2 & 1 & $x$ & & $x$ & & 20 & ACGIH & NR & - & 300 \\
\hline Limpiador de rodillos & $>5000$ & $>3160$ & $>1000$ & 5 & 4 & 3 & $x$ & & $x$ & & 100 & ACGIH & NR & - & NR \\
\hline Limpiador 4 & 11000 & 15251 & NR & 5 & 5 & - & & $x$ & & $x$ & 15 & NGV & 30 & NGV & NR \\
\hline Tinta de numeración & 5709 & 2 & $>0,85$ & 5 & 1 & 1 & & $x$ & & $x$ & 10 & INSHT & NR & - & NR \\
\hline Aceite & $>5000$ & $>2000$ & & 5 & 2 & 1 & & $x$ & & $x$ & 5 & OSHA & NR & - & NR \\
\hline Refrigerante & 4000 & 9530 & 2725 & 4 & 5 & 4 & & $x$ & & $x$ & 50 & OSHA & NR & - & 125 \\
\hline Tinta termocromática & $>5000$ & $>3160$ & $>1000$ & 5 & 5 & 4 & $x$ & & $x$ & & 100 & ACGIH & NR & - & NR \\
\hline Tinta de impresión & 1131 & $>1000$ & 0,37 & 2 & 2 & 1 & & $x$ & & $x$ & 10 & OSHA & NR & - & NR \\
\hline
\end{tabular}

Fuente: Hojas de seguridad de las sustancias, proporcionadas por la industria gráfica, nombre y marca de las sustancias no colocadas por protección de imagen. 